\title{
Improving Client Service Delivery using Standardized Nursing Care Plan: A Case Study of 37 Military Hospital
}

\author{
Article by Linda Appiah \\ SRN, CCN, PGD/NScN, BSc /Admin., Texila American University \\ E-mail: cutieappiah@gmail.com
}

\begin{abstract}
To determine the effect of standardized nursing care plan on the quality healthcare delivery. Using a deductive approach, the experiment would test the hypothesis 'If electronic health records (EHR) improve quality of health care, then the use of standardized (electronic) nursing care plans would improve client services in health delivery'. The experiment was conducted over 15 working days. Pre and postimplementation feedback from participants selected by cluster sampling was documented and analyzed. Cochran's modified formula was used to calculate 66 participants to reflect the total population of nurses who actually work on the wards and provide care for admitted patients' in the 37 Military Hospital. This cross section of the nursing population plan care and render health services on Medical and Surgical Wards that admit female and male clients and the Dialysis Unit that also plans care for acute and chronic patients of both genders. The study revealed that 58 (87.9\%) were of the opinion that LASNCP would be an effective strategy to promote use of nursing process, 59 (89.4\%) agreed an online version would help nurses to use care plan, and 62 (93.9\%) respondents agreed LASNCP can improve the quality of nursing care patients receive thus accepting the alternate hypothesis. Uptake and utilization of standardized nursing care plans to improve client service delivery is recommended.
\end{abstract}

Keywords: client service delivery; Standardized Nursing Care Plan; electronic health records; quality of health care.

\section{Introduction}

Nursing care plans (NCPs) help plan appropriate care needs for individual patients and also serve as a document that can help with auditing interventions rendered. Most importantly it is a communicative tool that helps the health team members interact effectively. Herold-Majumdar et al. in 2016 stated that health-care providers invest substantial resources for NCP and documentation. They recommend purchasing electronic or paper-based tools, and licenses as well as training nurses. Dawn Papandrea in January 2018 stated in her article that is to ensure all healthcare team members are receiving and planning for patient with same goals and no contradictions, a care plan is needed.

Bodeheimer and Sinsky (2014a) suggested that health team documenting part or all information into EHR as it has been associated with staff satisfaction, improved revenues and equipped them to better manage early discharged patients. Hence a well-structured Nursing Care Plan in paper base or an electronic format would enhance effective communication and promote quality health care delivery. This could also promote Nurses compliance to use the nursing process for the health needs of a patient.

Information and Communication Technology (ICT) is recommended by researchers that it improves quality of patient-centered healthcare care and educates health team members. They think if strategies are used to facilitate their implementation and their integration into nursing care, more benefits could be yielded and negative eliminated to support nursing care thereby improve patient outcomes. (Rouleau et al. 2015). 
DOI: 10.21522/TIJMG.2015.SE.19.01.Art002

ISSN: $2520-310 \mathrm{X}$

\section{Literature review}

A descriptive study on challenges associated with the implementation of the nursing process from 1970 to 2013 by Zamanzadeh et al in 2015 revealed several challenges. Their study revealed that Nurses lacked proper knowledge on the idea on how to implement the process. They also found lack of time to implement the process and the contrast between using the nursing process during clinical practice and when being taught in school. Other relevant challenges included time needed to obtain the earliest history of the patient to help formulate nursing diagnosis. Another challenge they found, was related to the process of recording steps of the nursing process, though some nurses agreed on the use of the nursing process being time consuming; it is reported that another study (Keenan et al. 2008, and Rouleau et al. 2017) showed influenced usage of the nursing care planning system when it was computerized.

With my experience as a Nurse Educator and widely published literature confirmations (Hagos et al. 2014), I have realised that student and ward nurses find it a challenge to develop nursing care plans and implement the nursing process during their clinicals to render care services. They are however assigned supervisors and guided to successfully use it during case study on patient care. Though the benefits are widely known and its usage compulsory for the partial fulfilment and award of a diploma certificate, when students complete their two (Nursing Assistant Clinical-NAC) or three years standard training programme as a Registered Nurse (RN), they hardly utilize the nursing care plan on the wards when employed. Nurses need to overcome these challenges and use this NCP, encompassed in the nursing process and other Evidence-Based Practices to improve nursing care rendered. Hence, if a standardized nursing diagnoses tool that is user friendly results in quality health care delivery, then the use of electronic nursing care plans would also be useful for improved client service delivery. Monsen et al. in 2011 published in their article that SCP's enabled a thorough assessment of the condition, gave appropriate description of goals and outcomes desired. Though their goals were to assess effectiveness of SCP and prevent readmission, they reported a large gap in use of NCPs which had published standards or guidelines as it did not provide solutions. They also found that EB-SCPs provide a shared standard for use in practice and future home care research and that the process could be an exemplar for development of EB-SCP's standards to be used for assessment and documentation to support global population health and research. Poder et $a l$ in 2015 said the use of SNCP could help Nurses comply to evidenced-based client care and thereby improve care and safety. They specifically concluded that electronic versions of the standardized care plan improved documentation of clients diagnosed with stroke within the first few days for stroke victims. In 2008, Dahm and Wadensten reported that majority of the Nurses surveyed believed that SCP's facilitate high-quality care and agreed would reduce time spent on documentation, though they felt the need for all nurses to be trained on how to use them. Jeff Rater in 2017 reported that standardised care plan must incorporate individual needs and limitations to actively involve patient in their care. He also stated that using standardized assessment and goals with the experiences of care giver would facilitate best practices for optimal client care. Bodeheimer and Sinsky in 2018b recommended 'Quadruple Aim' that is; 1. Improving the work life of health care providers, including clinicians and staff. 2. Improving the patient experience of care (including quality and satisfaction). 3. Improving the health of populations.4. Reducing the per capita cost of health care. As burnout of workforce would lead to dissatisfaction of patient and eventually increase cost. Therefore, health equity or achieving joy at work can be facilitated with tools (standardized nursing care plans) to ease planning and implementing care for patients. Leslie Small (2015) also advocates for quadruple aim for better health and lower cost.

As a practising Nurse Educator, I have the priviledge of hearing first hand complaints from trainee nurses about their difficulties in applying the nursing process (care plan) concept at the clinical area. The staff nurses catalogued their challenges in formulating Nursing Diagnosis, implementing interventions and documenting the nursing care plan at the wards. This challenge often inhibits their ability to adhere strictly to using the care plan as a standard tool for providing standardized individual nursing care. I understand the nursing care plan to be ideal to guide Nurses provide care for patients based on their health concerns needs or problems using a systematic scientific approach; in this case a Standardized Nursing 
Language (SNL) which provides special terms for clinical judgements realised during assessment of a client. This taxonomy provides continuity and communicates the wholistic care colaborated by nurses and clients to improve the quality of care rendered. However the SNL remains a challenge and though most needed nusing care are being rendered, the concept of the nursing diagnosis terminologies to be formulated momentarily and documented become the hindering blocks to students and some practioners alike.

The term (nursing diagnosis) is formulated based on the health problem and related to a cause or another health problem as they are rarely independent. For instance, a physiological problem of a system (risk for unstable blood glucose level) could be related to another system or a causative problem (insulin deficiency, deficit knowledge, and or sedentary activity). This then helps to identify and determine the appropriate intervention in order of priority. It is also true that, wrongly relating a problem would result in wrong intervention and subsequent poor outcome. This research involves 66 practising clinical nurses selected from clusters representing 13.75 percent of the total population of qualified nurses (480) in the hospital. Their input would be analyzed to facilitate the application of this new standardized care plan adopted from Gordon's functional health patterns developed in 1987 (Table 1). Their feedback would help determine if introducing standardized nursing care (appendix 1) and subsequently plan care would facilitate its easy usage at the clinical area and improve quality of care. This project could be duplicated worldwide to increase the utilization of nursing care plans at the clinical setting, provide reliable information and communicate health needs and interventions warranted. Above all, it would help in auditing nursing care as well as assessment, monitoring and evaluation of care rendered. These achievable goals would ultimately go to improve the level and quality of nursing care delivery to clients.

Table 1. Gordon's functional health patterns (nursing theories, Sept. 2013)

\begin{tabular}{|l|}
\hline 1. Health Perception Health Management Pattern \\
\hline 2. Nutritional Metabolic Pattern \\
\hline 3. Elimination Pattern \\
\hline 4. Activity Exercise Pattern \\
\hline 5. Sleep Rest Pattern \\
\hline 6. Cognitive-Perceptual Pattern \\
\hline 7. Self-Perception-Self-Concept Pattern \\
\hline 8. Role-Relationship Pattern \\
\hline 9. Sexuality-Reproductive \\
\hline 10. Coping-Stress Tolerance Pattern \\
\hline 11. Value-Belief Pattern \\
\hline
\end{tabular}

Gordon's functional health pattern also provides holistic care under eleven categories as tabulated in Table 1. The taxonomy II of NANDA nursing diagnosis is derived from Gordon's functional health pattern which was first developed in 1987 but since 2002 Nanda International (Nanda-I) continually revises standardized nursing diagnostic terminology to facilitate its usage.

The Nanda-I system of nursing diagnosis however provides for four different areas. First is the 'actual diagnoses. An example of which is: Sleep deprivation, depicting an existing finding found during a clinical judgment about human physiological responses to health conditions and life processes. The second area being a 'potential occurrence' because of human exposure to hazard and or increased susceptibility to respond adversely to exposed risk. An example being: Risk for shock. A third area Nanda-I also provides for is a 'health promotion diagnosis'. An example being, readiness for enhanced nutrition. This is also based on findings upon assessment that a person, family or community expresses the desire to improve their wellbeing with specific positive health behavior. The fourth area they formulate is 'syndrome diagnoses' where a clinical judgment describes a specific group of nursing diagnoses that may occur together and are best managed with similar interventions. An example being, Relocation stress syndrome. Yilmaz et al. in 2015 published Nanda-I nursing diagnosis (table 2) 
DOI: 10.21522/TIJMG.2015.SE.19.01.Art002

ISSN: $2520-310 \mathrm{X}$

determined by students during a study according to Gordons functional health showing four areas attended to. Values-beliefs and sexuality-reproduction nursing diagnosis were not formulated. The Omaha system amongst others also exists based on researches conducted in 1975 and 1993. It consists of: Problem Classification Scheme (client assessment); Intervention Scheme (care plans [no longer accepted] and services); Problem Rating Scale for Outcomes (client changelevaluation). It aims for easy data collection, documentation on health care team interventions, client strengths and needs to improve care.

Nursing Practice (ICNP); Nursing Minimum Data Set (NMDS); Nursing Intervention Classification (NIC); Critical Care classification (CCC); Nurse-Midwifery Clinical Data Set (NMCDS); Nursing Data Dictionary (NDD) (Monsen et. al 2011). These wide ranges of classifications and terminologies could lead to more confusing and varied standardization of care plans. Different standardised methods, terminologies and classifications exist for instance, International Classification for the lean sigma six methodology approaches would help develop a new standardized care plan. This methodology as explained by Grave in 2014 being: Define, Measure, Analyze, Design and Verify (DMADV) has a lot in common with the elements of the existing nursing process: Assessment, Diagnosis, Planning, Implementation and Evaluation (ADPIE) as with the Nanda-I nursing diagnoses process. In that Assessment, Diagnosis and Define attempt to explain the problems (define) faced in the situation from data gathered. Planning just like Measure and Analysis implies stating desired, achievable and realistic goals that can be measured and would be a solution to the problem. Implementation in ADPIE is to undertake or put into practise those measures planned that would best provide solutions and Design in DMADV. It also discusses available best methods analysed that would best solve the problems. Finally, Evaluation just like Verification is when desired goals are assessed to know if implemented plans or designs have been attained or there is need for modification. Bhaskar et al. in 2012 reported increased productivity and customer satisfaction and general improvement in all departments that use sigma six methodology to address their challenges.

Table 2. Nanda diagnosis

\begin{tabular}{|l|l|}
\hline $\begin{array}{l}\text { NANDA diagnosis determined by students according to } \\
\text { Gordon's functional health Patterns }\end{array}$ & N (\%) \\
\hline Health perception- management & $90(10.0)$ \\
\hline Risk for contamination & $18(2.0)$ \\
\hline Risk for falls & $15(1.7)$ \\
\hline Risk for bleeding & $9(1.0)$ \\
\hline Contamination & \\
\hline Nutritional- metabolic pattern & $57(6.4)$ \\
\hline Imbalanced nutrition -less than body requirements & $32(3.6)$ \\
\hline Excess fluid volume & $32(3.6)$ \\
\hline Nausea & $28(3.1)$ \\
\hline Impaired skin integrity & $21(2.3)$ \\
\hline Impaired oral mucous membrane & $18(2.0)$ \\
\hline Risk for impaired skin integrity & $13(1.4)$ \\
\hline Hyperthermia & $10(1.1)$ \\
\hline Deficient fluid volume & \\
\hline
\end{tabular}


Texila International Journal of Management

Special Edition Apr 2019

\begin{tabular}{|c|c|}
\hline Risk for impaired oral mucous membrane & $9(1.0)$ \\
\hline Interrupted breastfeeding & $8(0.9)$ \\
\hline Risk for imbalanced fluid volume & $6(0.7)$ \\
\hline Unstable blood glucose & $6(0.7)$ \\
\hline Imbalanced nutrition- more than body requirements & $4(0.4)$ \\
\hline Elimination & \\
\hline Constipation & $34(3.8)$ \\
\hline Diarrheal & $11(1.2)$ \\
\hline Risk for constipation & $9(1.0)$ \\
\hline Functional urinary incontinence & $6(0.7)$ \\
\hline Activity- exercise pattern & \\
\hline Activity intolerance & $60(6.7)$ \\
\hline Fatigue & $26(2.9)$ \\
\hline Total self-care deficit & $17(1.9)$ \\
\hline Ineffective breathing pattern & $17(1.9)$ \\
\hline Ineffective airway clearance & $17(1.9)$ \\
\hline Sleep- rest pattern & \\
\hline Disturbed sleep patterns & $61(6.8)$ \\
\hline Cognitive- perceptual pattern & \\
\hline Pain & $90(10.0)$ \\
\hline Deficient knowledge & $27(3.0)$ \\
\hline Acute confusion & $6(0.7)$ \\
\hline Self-sensing, self-concept pattern & \\
\hline Anxiety & $60(6.7)$ \\
\hline Disturbed body image & $16(1.8)$ \\
\hline Powerlessness & $14(1.6)$ \\
\hline Fear & $4(0.4)$ \\
\hline Role- relationship pattern & $8(3.6)$ \\
\hline Social isolation & $897(100)$ \\
\hline Sexuality- reproductive pattern ${ }^{*}$ & \\
\hline Coping- stress tolerance pattern & \\
\hline Ineffective coping & \\
\hline Value- belief pattern* & \\
\hline Other diagnoses ${ }^{* *}$ & \\
\hline Total & \\
\hline
\end{tabular}


DOI: 10.21522/TIJMG.2015.SE.19.01.Art002

ISSN: $2520-310 \mathrm{X}$

"These include risk for trauma, risk for vascular trauma, ineffective health management, risk for aspiration, risk for unstable blood glucose, ineffective tissue perfusion, impaired spontaneous ventilation, impaired cardiac output, impaired walking, sedentary lifestyle, self-neglect, impaired comfort, impaired memory, risk for loneliness, hopelessness, impaired verbal communication, interrupted family processes, and risk for suicide. Yilmaz, et al. (2015).

The aims of health care organizations worldwide include improving the quality of health care services to improve customer experience and achieve satisfaction. In the quest to improve health care and client service delivery, the introduction of electronic health record (EHR) and standardized tools promises to be beneficial worldwide. In coming up with this research proposal and measures taken to meet best practice and standard requirements, Sample Care Plans from the Cardiothoracic Unit and Surgical Department in the Korle Bu Teaching Hospital as well as that from the Intensive Care Unit of the 37 Military Hospital were understudied. These Sample Care Plans were found to be adopted from Scotland and South Africa and adapted to suit the Ghanaian setting. Online literature was reviewed to know the general trend and international best practice adopted in the design and types of care plans was also helpful. This experiment would employ the participation of clinical nurses and clinical supervisors to introducing a newly designed standardized care plan (Appendix 1). Their feedback would provide needed information and data. This would evaluate the hypothesis: If EHR will improve quality of health care delivery, then the use of standardized (electronic or paperbased) standardized nursing care plans will be useful for improving client services in health delivery'.

\section{Methodology}

\section{Study design}

As part of this evidence-based study, in collaboration with my supervisors and the clinical nurse practitioners I would develop a standardized nursing care plan which would be based on the Nanda-I and Gordon's functional health pattern and concepts adopted from care plan charts reviewed. The sample of Nurses for the study would be selected through cluster sampling (figure 1). The LAppiah Standardized Nursing Care Plan (LA SNCP) tool would be administered to randomly selected Nurses on selected specific wards in the 37 Military Hospital. They would be asked to use the tool to plan care for their admitted patients and a questionnaire also given for documentation with feedback from this experiment conducted to test the hypothesis and help answer questions this research study seeks to find.

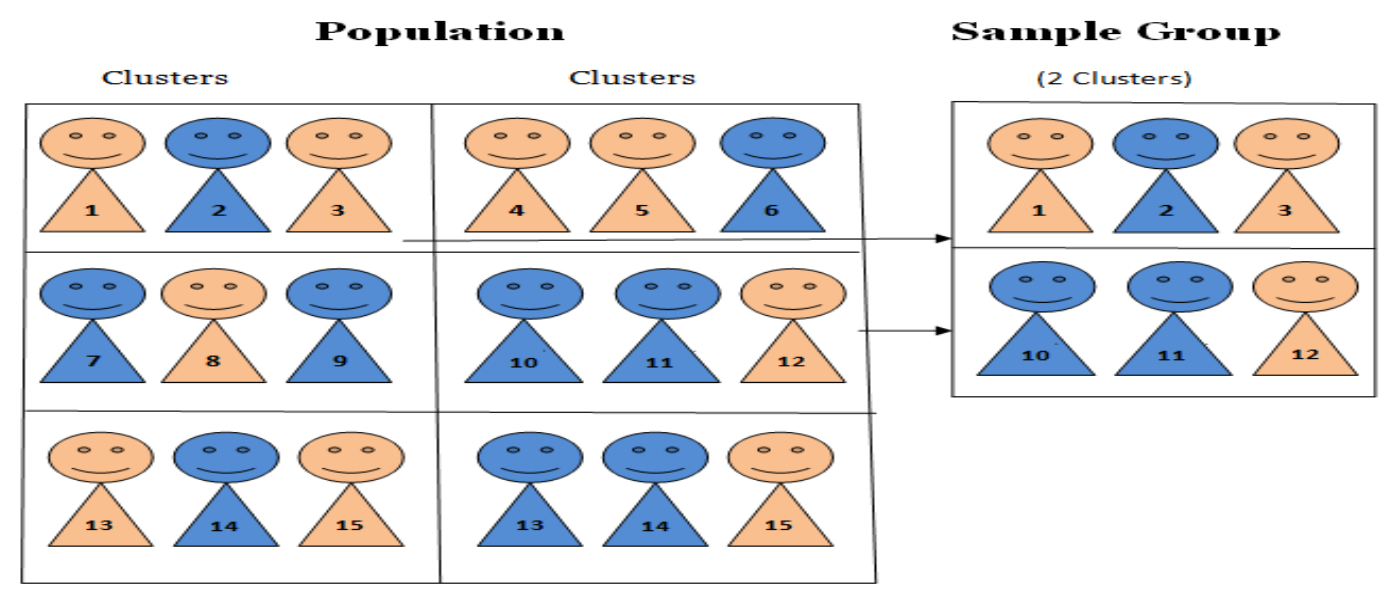

Figure 1. Cluster sampling

Retrieved from:

https://research-methodology.net/sampling-in-primary-data-collection/cluster-sampling/ 


\section{Data collection}

A questionnaire was also be used to collect additional data. During the experiment, a questionnaire was be given prior to the administration of a LASNCP (independent variable). The section ' $A$ ' and ' $B$ ' part of the questionnaire (Appendix 2) would be filled first prior to the experiment. This would help gather data on their professional status and bio-data, as well as their views on the existing nursing care plans. Data analysis was by Microsoft Excel xlsx. The LAppiah Standardized Nursing Care plans (Appendix 1) would then be introduced to the nurse participants with brief directives on how to use it. They would be directed to use the care plan for at least five working days. Views and compliance on use of the nursing care plans would be ascertained through their feedback that would be solicited from the administration of a questionnaire. Amendments or variations would be made to the SNCP and re-administered to plan care for another 5-10 days. - The section ' $\mathrm{C}$ ' of the questionnaire would then be filled by participants and feedback would be analysed to prove or disprove the research hypothesis: 'if the use of standardized (electronic or paper based) nursing care plans would improved client services in health delivery'.

\section{Target populations and study sample}

The sampling design process would be carried out with these five steps: population define; determination of sampling frame; selection of the sampling technique; determination of the sample size, and execution of the sampling process (Ragab, M. A. F. and Arisha A. 2017). The study would be conducted at the 37 Military Hospital on Nurses who in the clinical setting plan and provide care for patients admitted on the selected wards in the military Hospital. 'Bandoh A' and 'Bandoh B' (Medical Ward), 'Tamakloe Ward' (Surgical Ward), Dialysis Unit, 'Neuro Ward' (Surgical Ward), mixed ward (male and female) would be selected as the as sampling frame. These various categories selected (clusters); Wards and Units represented reflect the care-settings where admitted patients receive care. This process of cluster sampling reduces cost of the research and improves sampling efficiency levels. Primary data would be collected from the participants randomly selected from the groups of Nurses (in the selected wards) providing care in the clinical setting. Though cluster sampling may not reflect the diversity in the sampling frame and have higher sampling error than other techniques and it would help draw valid inferences from the general population.

\section{Sample size and sampling process}

The Medical and Surgical Wards, including the female and male representative Wards to present a cross section work area the nursing population plan care for patients, Dialysis Unit Nurses who render care to a mixed gender with varied conditions would also be sampled. Then random selection of participants (volunteers) in these selected clusters would be included in the study. Probability sampling is commonly used in cases of the questionnaire survey because it removes bias and maximizes the external validity. For reliable insights from the research study, an ideal sample size needs to be sampled.

A pilot study on 20 participants the greater number instead of $9 \%$ of projected final sample (65) that would have yielded 6 participants for pilot study, as explained in Cocks rule of thumb (M. Conroy, 2016). The pilot study was conducted to test the validity of the questionnaire and LAppiah Standardised Nursing Care Plan and slight amendments made to the tools. The Cochran formula allows you to calculate an ideal sample size to represent the population with desired levels of precision and confidence. It is however, considered especially appropriate when researching large populations, though a modified Cochran formula for sample size in smaller populations would be used. Taro Yamane's formula appears to be simpler for finite populations but outdated. The sample size was determined by. 
DOI: 10.21522/TIJMG.2015.SE.19.01.Art002

ISSN: $2520-310 \mathrm{X}$

\section{Cochran's formula}

$$
n_{0}=\frac{Z^{2} p q}{e^{2}}
$$

- Where e (margin of error) is the desire level of precision

- $\quad \mathbf{p}$ is the (estimated proportion of the population which has the attribute in question)

- $\mathbf{q}$ is $1-p$

As there is not much documented information on participants about their use of nursing care plans, a maximum variability population would be estimated as $50 \%(\mathrm{p}=0.5)$

For a $95 \%$ confidence level (gives $\mathrm{Z}$ values of 1.96 , per the normal tables), so that the probability of samples estimated, $95 \%$ could be expected to be included in the population parameter.

and at least $5 \%$ plus or minus error (e 0.05$)$.

therefore, sample data is $=\left[(1.96)^{2}(0.5)(0.5)\right] /(0.05)^{2}=385$.

As the total populations of nurses being sampled in the clusters are 77 a Cochrans modified formula to calculate for smaller sample size is used.

$$
n=\frac{n_{0}}{1+\frac{\left(n_{0}-1\right)}{N}}
$$

Where

no is Cochran's recommended sample size

$\mathbf{N}$ is the population size

$\mathbf{n}$ is new sample size.

Therefore, new sample size would be

$385 /[(1+(384 / 78)]=66$

The study period is estimated be from $30^{\text {th }}$ October to $22^{\text {nd }}$ November 2018 when permission is granted. Due to the nature of work shifts and timings, the tools would be made available for all shifts with simple and clear instructions attached, my telephone number and contact details if further clarification required. The actual target population will be nurses trained to use care plans.

\section{Inclusion criteria}

All Clinical Nurses including Orientation Nurses (staff nurses on rotation) on duty within the selected wards that volunteer to participate and give their consent would be included in the study sample.

\section{Ethical considerations}

Ethical approval will be obtained from the 37 Military Hospital Institutional Review Board and the Institutional Review Committee at the Texila American University, India.

Informed consent and permission will be obtained from Departmental heads and study participants before data collection. Data will be stored for five (5) years before destroyed by shredding. Quantitative data will be stored in a safety box under lock and key or on a password protected drive. Any other ethical issues or discomforts that may arise during study will be identified and discussed with involving participants and same amicably addressed with available resources and options.

\section{Findings}

The study on 66 Nurses on improving client service delivery using standardized nursing care plan (LASNCP) yielded results consistent with existing research findings and a good initiative to quality 
health care. The findings experiment conducted over 15 working days would be communicated through a publication of the survey undertaken in the Texila (institution's) Journal and a copy of study results and Standardized Care plan would be given to the In-Service Coordinator to adopt for the hospital if successful. Copies would also be given out to the Ghana Nursing and Midwifery Council (NMC) for publication and adaptation if validated. An opportunity would be sought to present it at the annual conference of the West African College of Nurses (WACN).

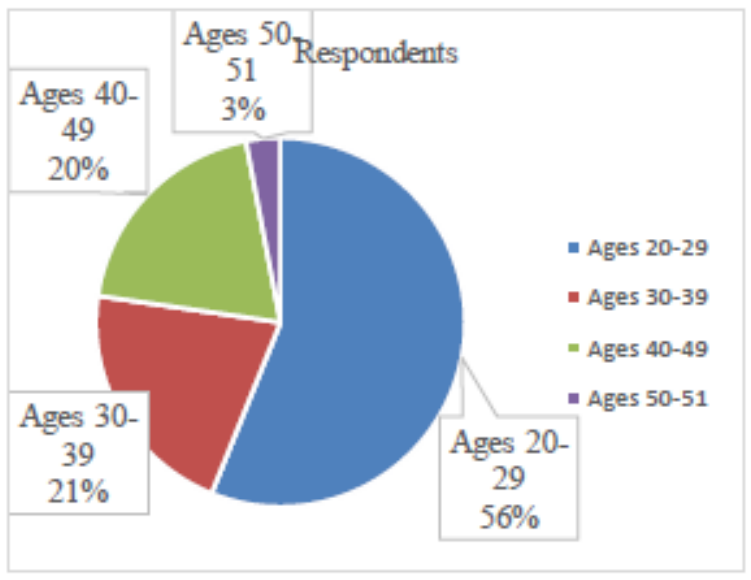

Figure 2. Age range of respondents

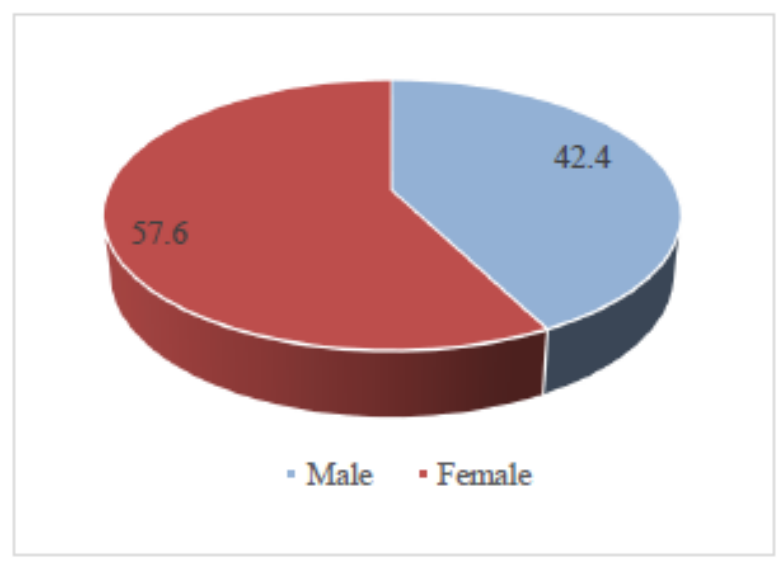

Figure 3. Gender

\section{Discussion on findings}

The findings are relevant for planning and improving services to client to attain client and nurse satisfaction. Experiment was conducted with 66 Nurses on use of LAPPIAH STANDARDIZED NURSING CARE PLAN consistent with NANDA-1 Taxonomy II and Gordon's functional health patterns. Prior to the main study, a pilot study was carried out with 20 Nurses to determine any possible difficulties in using the newly developed LASNCP tool and questionnaire. Majority (90.9\%) are practicing as general nurses. 
DOI: 10.21522/TIJMG.2015.SE.19.01.Art002

ISSN: $2520-310 \mathrm{X}$

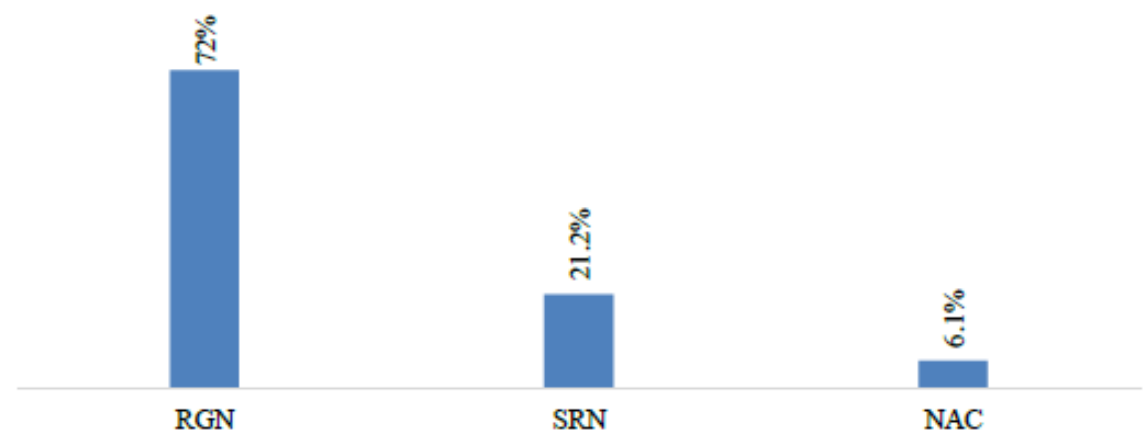

Figure 4. Grade of respondents

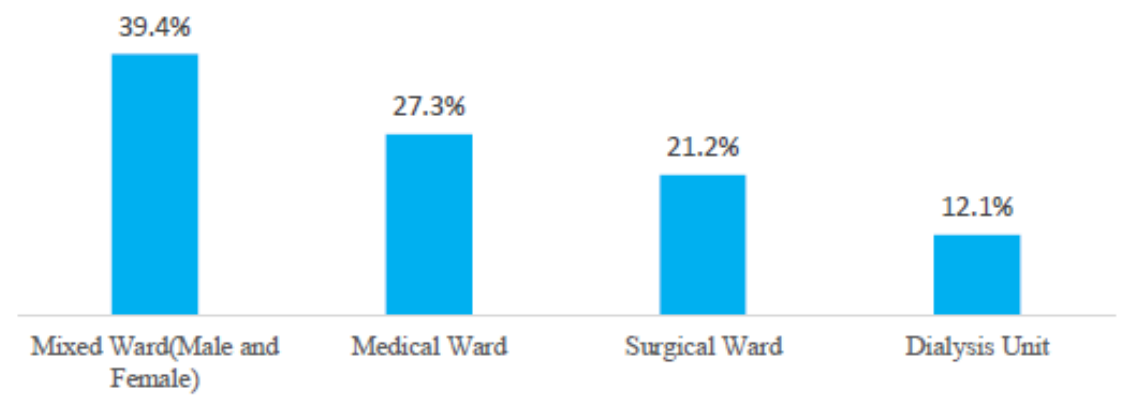

Figure 5. Current ward of practice

Minor amendments were made and from there the main study carried out. To reduce the effect of nonrespondents due to misplaced tools or delayed return of questionnaires, 75 questionnaires and care plan tools were printed and daily reminders through calls and visit to the wards were made to encourage them to use and provide feedback. A total sample size of 66 participants between the ages of 21- 51years was studied.

A total of $66(100 \%)$ participants' responses were analyzed. The majority of respondents (figure 2) were between the ages of 20-29 years representing 37 (56.1\%) participants with those in ages 30-39years being $14(21.2 \%)$ and 40-49 years being $13(19.7 \%)$ the two second highest group who voluntarily participated in the study with over 50 years being $2(3 \%)$. Interestingly $49(74.2 \%)$ of the respondents were Registered General Nurses and $64(90.7 \%)$ of respondents were practicing as General Nurses.

All $66(100 \%)$ participants thought there was a relationship between the nursing process and the quality of health care the patient receives and it also improves the care given to patients. Though $36(54.5 \%)$ respondents ticked that they use the nursing process in patient care, $12(18.2 \%)$ respondents ticked that there were guidelines on the use of the nursing process available at the clinical area.

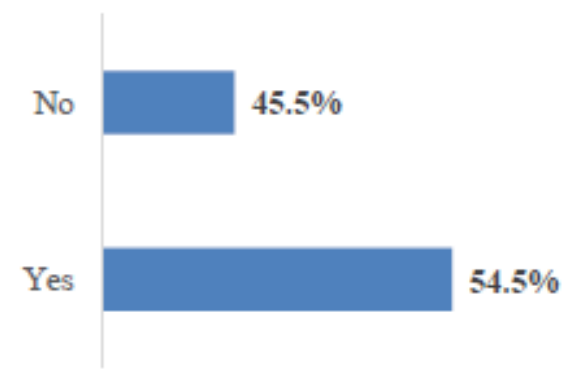

Figure 6. Usage of the nursing process in patient care 


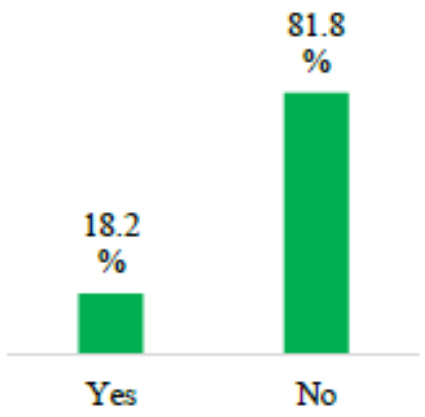

Figure 7. Availability of guidelines at the clinical area?

The percentage $(73.2 \%)$ responded that there are no strategies on the ward to promote use of the nursing process, is about the same percentage (77.3\%) also responded that there are on guidelines (fig 7 ).

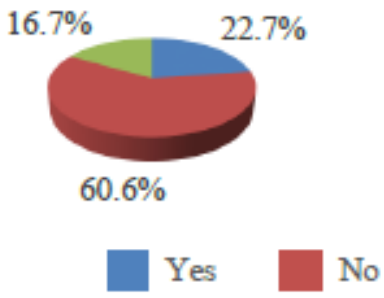

Figure 8. Are these strategies effective in promoting the use of the nursing process care plan?

After the administration and use of the LAppiah standardized nursing care plan (amended after the pilot study) on planning patient care, the 66 participants provided the following useful feedback. 64 (97\%) responded and $62(93.9 \%)$ answered the research question that the use of LASNCP would improve the quality of nursing care patient receive hence, improve client service delivery. However, 58 (87.9\%) had responded that the LASNCP would be an effective strategy to promote use of the nursing process care plan as shown in figure 9.

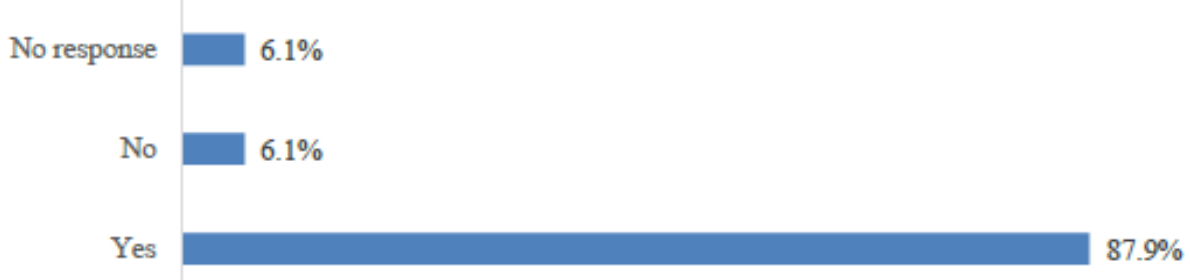

Figure 9. Would the LAPPIAH STANDARDIZED NURSING CARE PLAN be an effective strategy in promoting the use of the nursing process care plan 
DOI: 10.21522/TIJMG.2015.SE.19.01.Art002

ISSN: $2520-310 \mathrm{X}$

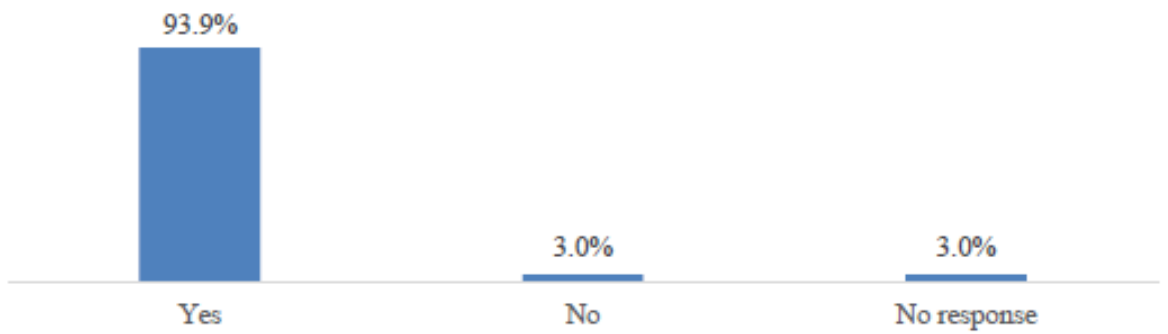

Figure 10. Would the LAPPIAH STANDARDIZED NURSING CARE PLAN improve the quality of nursing care the patient receives?

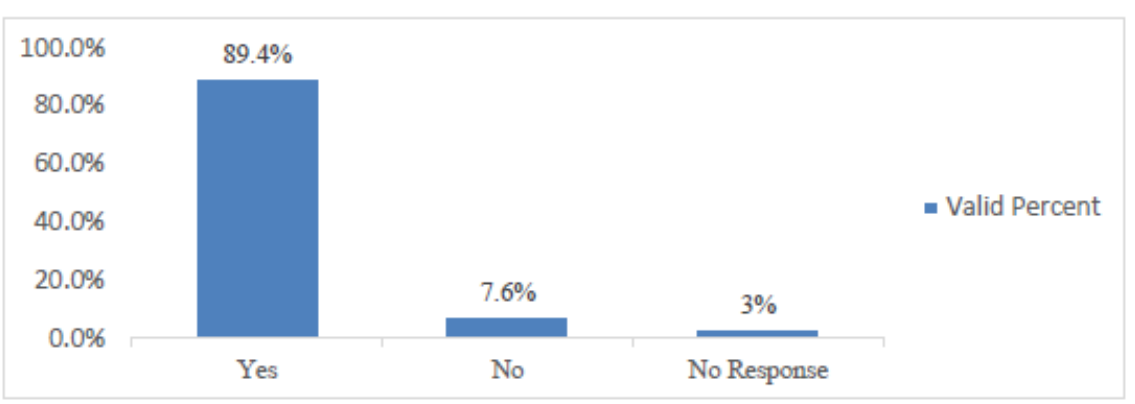

Figure 11. Will this format of 'LAPPIAH STANDARDIZED NURSING CAREPLAN' in an online version ehr help nurses in the use of nursing care plan?

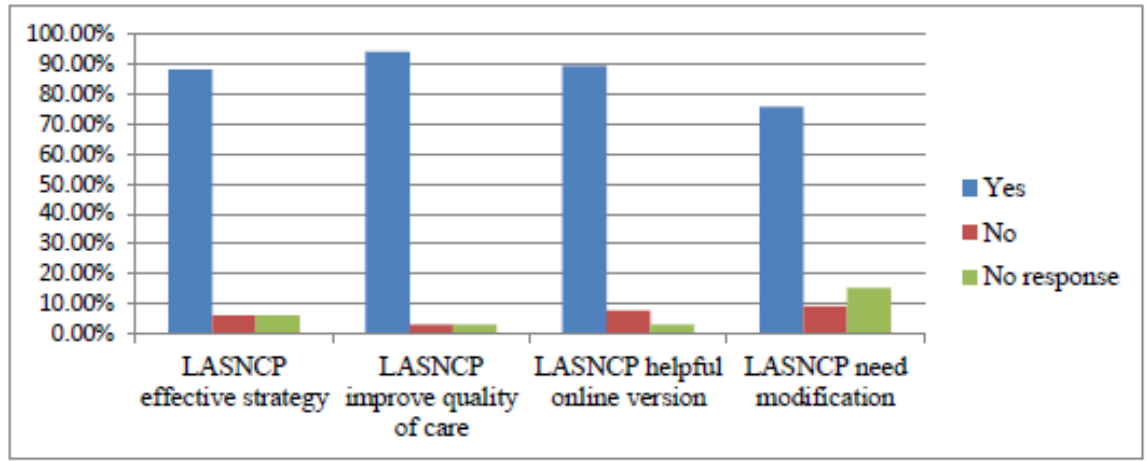

Figure 12. Comparism of results on 'LAPPIAH STANDARDIZED NURSING CAREPLAN'

This study revealed that $36(54.5 \%)$ out of the 66 respondents as depicted in figure 6 ticked that they use the nursing process in planning care for patients. This information is contrary to Agyeman-Yeboah, and Korsah (2018) findings that Nurses do not take the initiative in patients' care as professionals are expected to do and are subsequently unable to provide any better care to patients who on a specific day were not reviewed by their medical doctors. Implying that nursing care to patients was based on medical orders and nurse's discretion and not planned based on the specific needs of the patients. However, only $10(15.2 \%)$ respondents stated that there were strategies put in place to promote its utilization and 15 $(22.7 \%)$ respondents agreed the strategies were effective in promoting the use of the nursing process; care plan. Sixty-two (62) reflecting 96.9\% out of the 64 respondents that answered that the LAppiah Standardized Nursing Care Plan was easy to understand also agreed that it would improve the quality of nursing care that the patient receives. Other studies (Keenan et al 2008, and Rouleau et al 2017) reported that Nurses found it challenging to record the steps of the nursing process and that it was time consuming 
were the same findings made by Mutegi in 2016. However, there was increase in usage of the nursing care planning system when it was computerized. This supposes that LASNCP if made available through the Electronic Health Records would facilitate its use.

Respondents 62 (93.9\%) answered Yes to the question, 'would the LAppiah Standardized Nursing Care Plan improve the quality of nursing care the patient receives? were' and 59 (89.4\%) in figure 13 thought an online version accessible through Electronic Health Records will be of help to Nurses in the usage of the NCP were. Their response was consistent with Poder et al. (2015) who reported that the use of SNCP improved care and safety. Fifty (50) participants, recommended the modifications and summarized as follows;

- There should be an addition of more care needs or interventions especially for special conditions and areas; Paediatrics, Dialysis

- Though some wanted it less bulky and easy to use, they recommended spaces to be provided for additional nursing diagnosis, goals or interventions to be written by carer.

- To add more interventions; for instance, on risk of contaminations and bleeding under comfort [catheter care and changing soiled linen]; intervention for pre-operation teaching and familiarization to correspond to anxiety and knowledge deficit.

- Some thought it should be policy driven and more education on care plans usage.

- A small percentage wanted for the desired goals to be written as well as the evaluation columns to be with tick options because of time constraints.

\section{Clinical relevance}

If the above recommended modifications could be made to improve and facilitate the use of LA SNCP, it would support Bodeheimer and Sinsky (2014c) recommended 'Quadruple Aim' to improving the work life of health care providers, including clinicians and staff as it would make it easy to use and facilitate health service delivery which would result in Patient and Nurse satisfaction. Herold-Majumdar et al. in 2016 stated that, a resident's quality of life is affected by nurse's quality of life as a nurse in distress during working time experiences decrease in empathy and subsequently their understanding of the patient's experience and perspective.

\section{Conclusion}

The LAppiah standardized nursing care plan should be further developed and tailored to implement for use in the hospitals Electronic Health Records. I would also advocate for the use of Standardized Nursing Language with EHR to allow for ease of documenting nursing care and facilitate communication of nursing care across healthcare disciplines and healthcare environments. Johnson et al. (2016). Over 90\% responded positively to the utilization of the LASNCP to promote use of planning care and subsequently improve client service delivery as shown in figure 10 and 12. The null hypothesis was rejected and alternate hypothesis accepted. It is highly recommended that the development of effective NCP tools should be carried out under the guidance of researchers and Nurse Managers with focus on both users and clinical perspective on quality of life Herold-Majumdar A. et. al.2016. Now that it's been established that the LAppiah Standardized Nursing Care Plan is user friendly and acceptable to clinical nurses irrespective of their specialty, henceforth studies should be done to determine its effectiveness in improving care in the Ghanaian setting.

\section{Acknowledgement}

Sincere gratitude to Almighty God and the following:

- $\quad$ The Intensive Care Unit, 37 Military Hospital

- The Surgical Ward, Intensive Care Unit, Korle Bu Teaching Hospital

- The Cardiothoracic Unit, Korle Bu Teaching Hospital- Given a Glimpse of adopted Chart

- The Ethical Board 37 Military Hospital

- The Commanding Officer, 37 Military Hospital 
DOI: 10.21522/TIJMG.2015.SE.19.01.Art002

ISSN: $2520-310 \mathrm{X}$

- The Principal, Nursing and Midwifery Training College, 37 Military Hospital

- The Deputy Nursing and Midwifery Training College, 37 Military Hospital

- Dr. Gordon Appiah, Head of Neuro Surgery 37 Military Hospital

- Dr. Mary Ani- Amponsah of University of Ghana, Legon

- Major Rtd Don Chebe

- Mr Edmund Wiredu of Emetron Technologies

\section{References}

[1]. Agyeman-Yeboah, J., Korsah, K. A., \& Okrah, J. (2017). Factors that influence the clinical utilization of the nursing process at a hospital in Accra, Ghana. Online journal of BMC Nursing 16 (30). Retrieved from https://doi.org/10.1186/s12912-017-0228-0.

[2]. Agyeman-Yeboah, J. \& Korsah, K. A. (2018). Non-application of the Nursing Process at a Hospital in Accra, Ghana: Lessons from Descriptive Research. Online journal of BMC Nursing. Retrieved from: https://bmcnurs.biomedcentral.com/articles/10.1186/s12912-018-0315-x.

[3]. Ballantyne, H. (2016). Developing Nursing Care Plans. Online journal of Nursing Standard. 30(26) 51-60. doi: 10.7748/ns.30.26.51. s48.

[4]. Bhaskar, R., Kapoor, A., \&Au Vo, A. (2012). Pioneering the Health Care Quality Improvement in India Using Six Sigma: A Case Study of a Northern India Hospital. Online journal of Journal of Cases on Information Technology 14(4):41-55. doi: 10.4018/jcit.2012100104.

[5]. Bodenheimer T., \& Sinsky, C. (2014). From Triple to Quadruple Aim: Care of the Patient Requires Care of the Provider. Online Journal of Annals Family Medicine, 12(6), 573-576. doi: 10.1370/afm.1713.

[6]. Conroy. R. M. (2016). The RCSI Sample Size Handbook a Rough Guide. Retrieved from: http://www.rcsi.ie/files/research/docs/20160811111051_Sample\%20size\%202016.pdf.

[7]. Cluster Sampling - Research-Methodology. Retrieved from: https://research-methodology.net/sampling-inprimary-data.../cluster-sampling/.

[8]. Dawn, Papandrea (January 2018). Nursing Care Plans: What You Need to Know. Retrieved from: https://nurse.org/articles/what-are-nursing-care-plans/.

[9]. Dahm, M. F. \& Wadensten, B. (2008). Nurses' experiences of and opinions about using standardised care plans in electronic health records--a questionnaire study. Online journal of Journal of Clinical Nursing. 17(16): 21372145. doi: 10.1111/j.1365-2702.2008.02377. x.

[10]. Frater, J. (2017). Standardized Versus Personalized Care Plans Retrieved from: https://www.cmsatoday.com/2017/10/04/standardized-versus-personalized-care-plans/.

[11]. Gordon's 11 Functional Health Patterns. Retrieved from: https://www.bartleby.com/essay/Gordons-11Functional-Health-Patterns-FK6GBFPMYRPA.

[12]. Hagos, F., Alemseged, F., Fikadu Balcha, F., Berhe, S. \& Aregay, A. (2014). Application of Nursing Process and Its Affecting Factors among Nurses Working in Mekelle Zone Hospitals, Northern Ethiopia. retrieved from: https://www.ncbi.nlm.nih.gov/pmc/articles/PMC3933400/.

[13]. Herold -Majumdar, A., Schaller, M., Flescher, S., \& Behrens, J (2016). Effects of Nursing Care Planning Tools on Nurses' and Residents' Quality of Life in Long-Term Care Facilities: A Literature Review. Online journal of Journal of Nursing Education and Practice 7(4). doi: 10.5430/jnep.v7n4p32.

[14]. Johnson L., Karen-Leigh, E. \&, Giandin, J., A. (2018. A Systematic Literature Review of Accuracy in Nursing Care Plans and Using Standardized Nursing Language. Online journal of Collegian, 25(3) 355-361. doi: https://doi.org/10.1016/j.colegn.2017.09.006.

[15]. Jakobsson, J \& Wann-Hansson, C. (2012). Nurses' Perceptions of Working According to Standardized Care Plans: A questionnaire study. Retrieved from: https://onlinelibrary.wiley.com/doi/pdf/10.1111/scs.12009.

[16]. Keenan, G. M., Yakel, E., Tschannen, D. \& Mandeville, M. (2008). Patient Safety and Quality: An EvidenceBased Handbook for Nurses. Chapter 49Documentation and the Nurse Care Planning Process. Retrieved from https://www.ncbi.nlm.nih.gov/books/NBK2674/. 


\section{Texila International Journal of Management Special Edition Apr 2019}

[17]. Pöder, U., Dahm. M. F., Karlsson, N., \& Wadensten, B. (2015). Standardised care plans for in hospital stroke care improve documentation of health care assessments. Online journal of Journal of Clinical Nursing.24 (19-20): 2788-2796. doi: 10.1111/jocn.12874.

[18]. Rouleau, G., Gagnon, M.P., Côté, J., Payne-Gagnon, J., Hudson, E. \& Dubois, C. A. (2017). Impact of Information and Communication Technologies on Nursing Care: Results of an Overview of Systematic Reviews. Online journal of Journal of Medical Internet Research 19(4) retrieved from: https://www.jmir.org/2017/4/e122/.

[19]. Small, L. (2015, June). Why healthcare organization must embrace the 'Quadruple Aim'. Fierce Healthcare. Retrieved from: https://www.fiercehealthcare.com/healthcare/why-healthcare-organizations-must-embracequadruple-aim.

[20]. Yilmaz, F. T., Selma Sabanciogullar, S. \& Aldemir, K. (2015). The Opinions of Nursing Students Regarding the Nursing Process and Their Levels of Proficiency in Turkey. Online journal of Journal of Caring Science. 4(4): 265-275.doi: [10.15171/jcs.2015.027]. 